\title{
A sheep in wolf's clothing? Wild-type P53 disguises as mutant to promote leukemogenesis
}

\author{
Margaret A. Ferris ${ }^{1}$ and John S. Welch ${ }^{2}$ \\ ${ }^{1}$ Department of Pediatrics, Washington University and ${ }^{2}$ Department of Internal Medicine, Washington University, St Louis, MO, USA \\ E-mail: jwelch@wustl.edu \\ https://doi.org/10.3324/haematol.2022.280671
}

In this issue of Haematologica, Tuval and colleagues ${ }^{1}$ describe misfolded P53 (pseudo-mutant P53) as a marker of preleukemic cells in patients with DNMT3A-mutated acute myeloid leukemia (AML). TP53 is mutated across human cancers, including AML. P53 functions to protect genomic stability; interestingly, this can be achieved by activating distinct pro- and anti-apoptotic pathways. To add to this complexity, multiple TP53 isoforms can be expressed and post-translational modifications further influence P53 function within specific cellular contexts. ${ }^{2}$ Thirty years ago, Gannon and colleagues identified a monoclonal antibody (PAb240) that distinguished mutant P53 from wild-type, and theorized that this antibody recognized an epitope that was protected in the wild-type conformation. ${ }^{3}$ Zheng and colleagues later found a series of TP53 wild-type AML cell lines that express P53 in the mutant conformation, referred to as "pseudo-mutant P53". Since then, the pseudo-mutant conformation has been observed in alternative splice forms of wild-type P53, and these can decrease MDM2 binding, preventing degradation of activated P53. ${ }^{5}$ The current study expands on these findings, comparing expression of this pseudomutant conformation in different leukemia subpopulations in patients without TP53 mutations.

AML is a clonal disease associated with subclonal heterogeneity within individual patient samples. Two separate models of leukemic hierarchy have emerged. Mutation analysis suggests the presence of a founding clone, subsequent cooperating mutations in subclones, ${ }^{6}$ and a related preleukemic state, clonal hematopoiesis $(\mathrm{CH})$, associated with clonal mutations but retained normal hematopoietic maturation. ${ }^{7}$ Second, immunophenotypic analysis separates hematopoietic stem cells from progenitors and from more mature cells. Recent studies have sought to harmonize these two models, demonstrating that within a patient, immunophenotypic preleukemic-hematopoietic stem/progenitor cells (preLHSPC) can be identified with driver mutations (DNMT3A) but not cooperating mutations found in the AML blasts (e.g., NPM1c); in contrast to leukemic blasts, these preLHSPC retain their capacity for multi-lineage differentiation. Xenograft experiments show that these preL-HSPC have a growth advantage over non-mutated HSPC. ${ }^{8}$ Somatic TP53 mutations have been found at the preL-HSPC stage in $\sim 20 \%$ of cases, conferring a selective advantage in xenograft models. ${ }^{9}$
The current study examines P53 protein conformations in preL-HSPC from AML patients. Importantly, the use of AML cases with DNMT3A and NPM1 mutations again allowed for separation of leukemic blasts (both mutations present) from preL-HSPC (DNMT3A-mutated, NPM1 WT). The authors then assessed P53 for pseudo-mutant conformation. Similar to the findings in AML cell lines, ${ }^{4}$ Tuval and colleagues found expression of the pseudomutant P53 in TP53 wild-type primary AML patient samples. Using mass cytometry, the samples were further separated into leukemic blasts (immunophenotype defined at diagnosis) and preL-HSPC (CD34+CD33-CD15-CD11bCD19-CD79b-CD3-CD16-CD45RA-), and the ratio of pseudo-mutant to wild-type conformation P53 (PM/WT$\mathrm{CR}$ ) was examined in each individual cell. Interestingly, heterogeneity in P53 conformations was identified: within blasts the wild-type conformation was dominant (PM/WT$C R=0.53$ ), but in the less abundant preL-HSPC, the pseudo-mutant confirmation was enriched (PM/WT-CR = 3.06) (Figure 1). The high PM/WT-CR appears specific to the preL-HSPC and was not observed in normal stem cells (cord blood PM/WT-CR $=1.22$ ) or cells from a patient with DNMT3A-mutated clonal hematopoiesis (DNMT3 $A^{\mathrm{R} 882 \mathrm{H}}-\mathrm{CH}$ $\mathrm{PM} / \mathrm{WT}-\mathrm{CR}=0.53$ ) (Figure 2).

The authors go on to use a xenotransplant model to determine whether the high PM/WT-CR leads to a selective

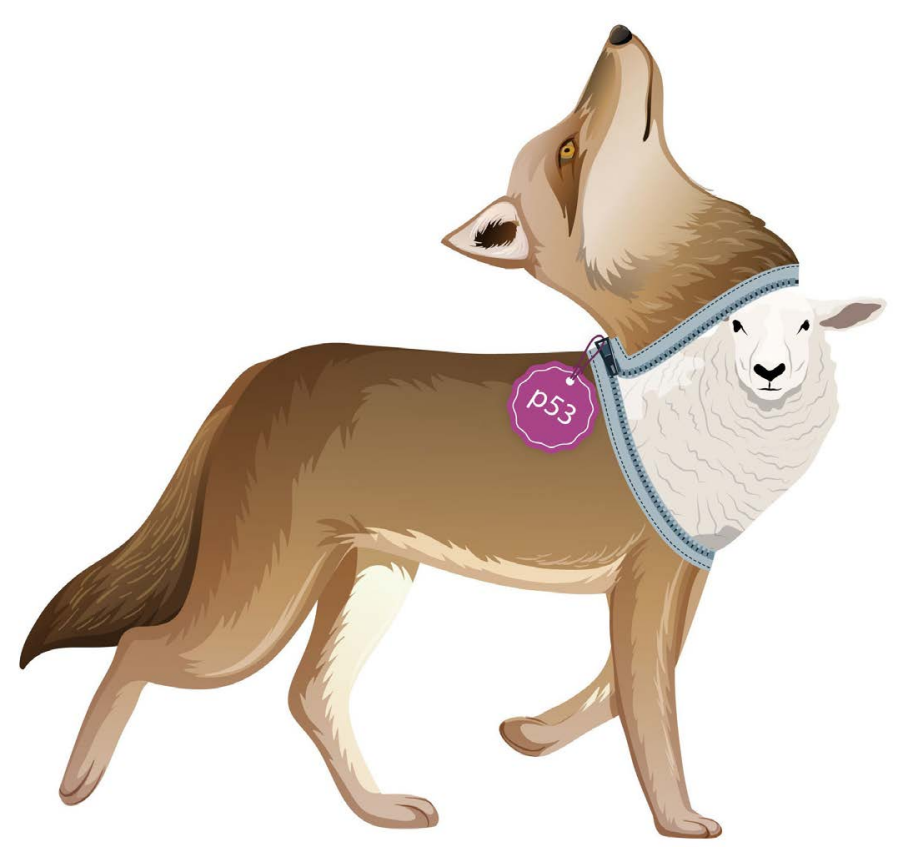

Figure 1. In this issue Tuval and colleagues demonstrate that wild-type TP53 may take on folding patterns similar to mutant TP53 (pseudo-mutant), and this may contribute to leukemic transformation. 
growth advantage of the preL-HSPC. The variability of engraftment potential of patient-derived xenografts limited the power of these studies as only one of the nine AML samples lead to engraftment of preL-HSPC (\#160005); the other samples engrafted the immunodeficient mouse marrow with leukemic blasts or non-leukemic stem cells (DNMT3A WT). Tumor \#160005 had one of the more modest PM/WT-CR ( 1.3). However, treatment with a P53stabilizing peptide, pCAP-250, decreased the engraftment potential of the preL-HSPC, but not of the non-preLHSPC, suggesting that the balance of wild-type and pseudo-mutant P53 contributed to the engraftment and expansion capacity of the preL-HSPC (Figure 3). Single cell RNA sequencing analysis of engrafted cells showed pCAP-250 treatment was associated with a reduction in specific subsets of cells. However, mass cytometric analysis of the engrafted cells did not correlate PM/WTCR level on susceptibility to PCAP-250, suggesting heterogeneity of pCAP-250 effects or pseudo-mutant P53 dependency that remain uncharacterized (Figures 5 and 6). Therefore, P53 may contribute to early transformational programs through both mutant and non-mutant effects. TP53 missense mutations are present in clonal hematopoiesis patients, ${ }^{10}$ are expressed at the preL-HSPC stage, and are associated with expressed proteins that alter sensitivity to chemotherapy and early transformation. ${ }^{9}$ Likewise, during transformation of induced pluripotent stem cells, overexpression of the $\Delta 133 p 53 \alpha$ isoform inhibits wild-type P53-inducible cellular senescence pathways, augmenting the reprogramming capacity. ${ }^{5}$ Now Tuval and colleagues demonstrate the heterogenous presence of pseudo-mutant P53 protein in TP53 wild-type hematopoietic cells, and the potential of pseudo-mutant P53 to influence transformation potential in preL-HSPC, distinguishing them from cells of clonal hematopoiesis where pseudo-mutant P53 does not appear dominant or active.

These provocative findings are thus far limited to small sample sizes and within DNMT3A-mutant/NPM1-wild-typedefined preL-HSPC, which were cleverly chosen to distinguish preL-HSPCS from leukemic blasts. Additional cases and types of mutations in preL-HSPCs will need to be examined to further define the frequency, distribution, and phenotypes of pseudo-mutant P53 in hematopoietic transformation. Knowing that P53 can have anti- and proapoptotic effects and that non-R882 DNMT3A mutationdriven $\mathrm{CH}$ is less likely to progress to $A M L$, it will be interesting to determine the distribution and phenotypes of pseudo-mutant P53 in additional forms of preL-HSCP and $\mathrm{CH}$. The mechanisms enabling pseudo-mutant folding in hematopoietic cells also remain uncharacterized, although splice variation and posttranslational modifications have influenced P53 folding patterns in other cellular contexts. This study serves as a starting point for defining the role of wild-type P53 modifications in leukemogenesis, and suggests that in the right context, wild-type P53 might put on wolf clothing to unexpectedly contribute to leukemic transformation.

\section{Disclosures}

No conflicts of interest to disclose.

\section{Contributions}

MAF and JSW wrote the manuscript.

\section{Funding}

JSW receives research funding from Janssen Pharmaceuticals and from Notable Labs.

\section{References}

1. Tuval A, Brilon $\mathrm{Y}$, Azogy $\mathrm{H}$, et al. Pseudo-mutant P53 as a targetable pheneotype of DNMT3A-mutated pre-leukemia. Haematologica. 2022;107(11):2548-2561.

2. Anbarasan T, Bourdon JC. The emerging landscape of p53 isoforms in physiology, cancer and degenerative diseases. Int $\mathrm{J}$ Mol Sci. 2019;20(24):6257.

3. Gannon JV, Greaves R, Iggo R, Lane DP. Activating mutations in p53 produce a common conformational effect. A monoclonal antibody specific for the mutant form. EMBO J. 1990;9(5):1595-1602.

4. Zheng A, Castren K, Säily M, Savolainen ER, Koistinen P, Vähäkangas K. p53 status of newly established acute myeloid leukaemia cell lines. Br J Cancer. 1999;79(3-4):407-415.

5. Fujita K. p53 isoforms in cellular senescence- and ageingassociated biological and physiological functions. Int $\mathrm{J}$ Mol Sci. 2019;20(23):6023.
6. Welch JS, Ley TJ, Link DC, et al. The origin and evolution of mutations in acute myeloid leukemia. Cell.

2012;150(2):264-278.

7. Jaiswal S, Fontanillas P, Flannick J, et al. Age-related clonal hematopoiesis associated with adverse outcomes. N Engl J Med. 2014;371(26):2488-2498.

8. Shlush LI, Zandi S, Mitchell A, et al. Identification of preleukaemic haematopoietic stem cells in acute leukaemia. Nature. 2014;506(7488):328-333.

9. Lal R, Lind K, Heitzer E, et al. Somatic TP53 mutations characterize preleukemic stem cells in acute myeloid leukemia. Blood. 2017;129(18):2587-2591.

10. Chen S, Liu Y. p53 involvement in clonal hematopoiesis of indeterminate potential. Curr Opin Hematol. 2019;26(4):235-240. 